\title{
Humanized Mandarin E-Learning Based on Pervasive Computing
}

\author{
Yue Ming, Zhenjiang Miao \\ Institute of Information Science, \\ Beijing JiaoTong University, Beijing 100044, P.R. China \\ Email: myname35875235@126.com
}

\begin{abstract}
E-Learning environments designated to facilitate long-distance learning are gaining popularity. Pervasive computing has a great potential for many next-generation IT applications. This paper describes pervasive computing system design and implementation for Mandarin e-learning. We propose a Human-centered Pervasive Computing System Model (HPC) and Layered Architecture Analysis and Design Method (LAAD). Based on the HPC model and LAAD method, a pervasive computing based Mandarin elearning system is designed and implemented. Its design and implementation issues are discussed in details.
\end{abstract}

\section{Introduction}

The impact of technologies has brought in many changes in the field of on-line education. The spectacular development of Internet provides motivation for studying the impact of new technologies on a natural area of education like e-learning. But our investigation indicates that the teaching systems of e-learning are lack of intelligence. Those systems cannot provide learners with personalized services.

As a result, we consider merging the e-learning system with pervasive computing platform. The "any- time, anywhere, and human-centered" feature of pervasive computing makes it a perfect technology for e-learning [1].

With Chinese opening and fast economy development in recent years, the communication between China and the world becomes more and more important in a wide range. So Mandarin, the important communication tool and culture carrier that lets the foreign country know China, attracts more and more governments, educational organizations and corporations. In order to facilitate the foreign learners, 
we design a pervasive computing Mandarin e-learning system and hope this system can make Mandarin learning conveniently and efficiently. In our work, we synthetically apply Human-centered Pervasive Computing System Model (HPC) and Layered Architecture Analysis and Design Method (LAAD) [2].

In section 2, we introduce the HPC and LAAD and describe the system requirements of a pervasive computing based e-learning system. Section 3 presents speech and tone recognition methods and the process of speech synthesizer for virtual audio teacher. Section 4 describes e-learning and m-learning system, especially the use of m-learning. Section 5 introduces personalized agent as the main advantage of our system. In section 6, we give the structure of IP-to-PSTN gateway and discuss the server. Finally, we describe the whole system design and implementation.

\section{System Model and Its Human Core Layer: System Requirements}

To consider key pervasive computing components as a whole, we propose the following Human-Centered Pervasive Computing System Model in [1]. This model can be illustrated in Fig.1.

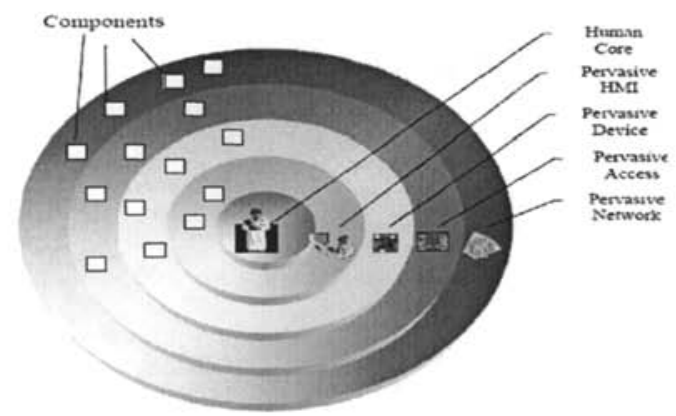

Fig.1 Human-centered Pervasive Computing system model (HPC)

We basically consider a pervasive system as five layers: Human/system requirements are major issues considered in Human core layer based on various different applications; Pervasive HMI layer considers the human and pervasive device natural interaction issues; Pervasive Device layer is for all devices human core user directly or indirectly interacts no matter whether they are visible or invisible; Pervasive Access layer is the bridge to connect the pervasive user device to the network environment; Pervasive Network layer as the system environment is the basis for pervasive computing system, which includes all the networks we can name today and future as long as the network connects to the internet directly or indirectly.

For a real pervasive computing system development, we have to analyze the application's requirements first and then design the system to meet the requirements. To mapping that to the HPC model, the requirement analysis is in the Human Core Layer. We call it Layered Architectural Analysis and Design method (LAAD). 
In our system, we consider many issues such as how to recognize and evaluate the utterance of foreign learners as follows:

-For beginner, our system will deliver prepared easy content to students, and ensure that the content reaches them anytime no matter where they are.

-It brings in feedback and ensures students to hear natural pronunciation.

-For outstanding learners with great fluency, the evaluation was executed with a brief quiz offered at the end of the lecture.

-If the learner feels tired, the system can make studying more interesting by numerous means like hearing/seeking someone, arranging a cartoon or game, etc.

The prominent advantages of our system: teach students in accordance with their aptitude (humanizing) and condition with flexible time and space. The system also facilitates communication between the teacher and the students.

\section{Pervasive HMI Layer: Speech and Tone Recognition, Speech Synthesizer for Virtual Audio Teacher}

The components in this layer are Context Awareness, Computing Paradigms for HMI, Multi-modal interaction, User Interfaces, etc [1, 2].

Fitting to the difference of each learner's timbre, the speech recognition should be speaker-independent Mandarin speech recognition.

Our speech recognition component is firmly based on the principle of statistical pattern recognition [3]. When students' utterance inputs into system, a front-end signal processor with a sequence of acoustic vectors converts the speech waveform and the language model computes its probability. For each phone there is a corresponding statistical model called a hidden Markov model (HMM). The sequences of HMMs needed to represent the postulated utterance are concatenated to form a signal composite model and the probability of that model generating the observed sequence is calculated.

Mandarin is tonal language. The Mandarin four different tones include a lot of important information. So we process the tone recognition separately. The pitch contrail can distinguish four Mandarin tones efficiently, and then an event detection pitch detector based on the dyadic wavelet transform which can detect the catastrophe point of speech signal when people speak [4].

One of the main barriers to on-line distance education is the feeling of alienation and isolation reported by students. Developing a sense of community among students is one of the critical factors in changing from lecturer-centered to student-centered approaches in the mode of teaching and learning. Presently, corpus-based Mandarin TTS component consistently produces the most natural and pleasant synthesized voice. Our system also has the TTS function module used as system virtual teacher.

With a view to natural speech, we built a large Mandarin text-to-speech corpus [5]. It is designed for both statistical prosody modeling, and context dependence of phonemic features. This approach may ensure the concatenated voice to have high intelligibility as well as naturalness of intonation. During the course of learning, the foreign learners are able to hear the "professor" anywhere, anytime and at any place. 


\section{Pervasive Device Layer: E-learning and M-learning}

This layer is mainly referred to the user interacted devices and related software. The components are Sensors and Actuators, Smart devices, various user devices such as PDA, Embedded System OS, etc [1, 2].

Complete independence of both location and time is often emphasized as the main advantage of e-learning. However, in traditional e-learning the minimum hardware and software requirements are still a Personal Computer (PC) and the HTML language of the Web. Consequently an absolute independence in location is not providing. These independencies are still not fulfilled with notebook, because a real independency in time and location means learning wherever and whenever a person wants to have access to learning material. Mobile learning (m-learning) is the next generation of e-learning and is based on mobile devices and the WML language.

M-learning can be a wide range of educational activities on PDA and mobile phone: Mandarin vocabulary lessons via mobile phone email; 'just-in-time' administration (scheduling, study prompts, and reminders) via mobile phones; language-oriented problem-asked learning modules on PDA; and recorded Mandarin listening materials accessed on mobile phones. They show that the unique combination of features in mobile devices - portability, connectivity, and low cost.

Mobile devices perform the functions of desktop computers, with the advantages of simplicity and improved access. But three limitations prevent mobile devices from replacing desktop PCs: bandwidth, running costs, and text input speed.

\section{Pervasive Access Layer: Personalized Agent}

The components in this layer are context awareness and reasoning, service management, etc $[1,2]$. We use a framework [6] to design and maintain language curricula (Fig.2), which advocates continuously evaluating needs, objectives, tests, materials, and teaching. We focus on evaluation, teaching and materials, including mobile hardware and software.

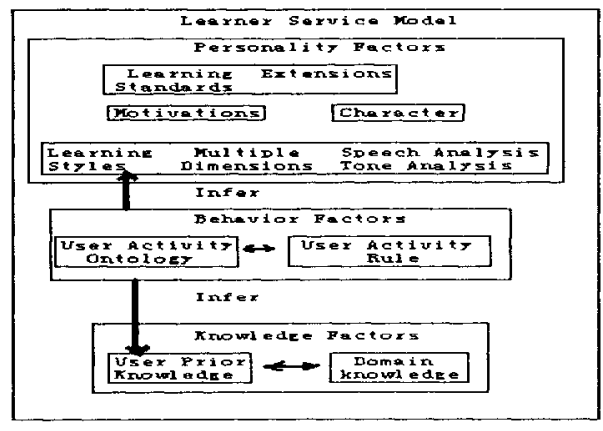

Fig.2 Conceptual design of learner model 
It is suitable to design a personalized agent for this layer which is typically composed of the learner service model depicted as Fig.2. The learner model is composed with three sub-categories: Personality Factors, Knowledge Factors and Behavior Factors which are inferable from elements of behavior factors in the Fig. 2 .

The Personality Factors are composed of four groups of elements. Learning standards elements contain dimensions such as Personal, Preference, and Portfolio, etc. They are coupled with extensions features so that distributed e-learning systems are the summation of mentality requirements, which affected not only the style of interaction, but also the style of behavior in learning (speech and tone).

The Knowledge Factors are composed of learner prior knowledge and domain knowledge. The domain knowledge represents the view of the e-learning systems. The learner prior knowledge represents the concepts associated with learning materials which have been provided by distributed e-learning systems to the learner.

Personality Factors and knowledge Factors are updated as new data for behavior factors are received. Data mining techniques can find the association rules [7].

In our system, the context sensed and used by the agent contains time and spatial contexts, and learner preferences profile. The use of context information (e.g. location, time, and mental states, etc.) has significant potential to simplify the learner's interaction with a complex system. If there is an agent who knows learner's studying level, it can help to find a lesson that satisfies what he needs automatically.

Personalized agent makes e-learning become intelligent, which would provide personalized knowledge service for learners and improve the learning quality greatly.

\section{Pervasive Network Layer: IP-to-PSTN gateway and Server}

This layer is mainly referred to the network hardware infrastructure and related software modules. The components are Gateways, Servers, Network Resource etc [1].

Today, with the expansion of the Internet, voice services are being provided increasingly with packet-switching networks based on Internet protocol (IP) technologies, i.e., voice-over-IP (VoIP). To realize complete voice calls between endpoints in the Internet and ones in the PSTN, VoIP service providers use IP-toPSTN gateways, which serve as bridges between the PSTN and IP networks [8].

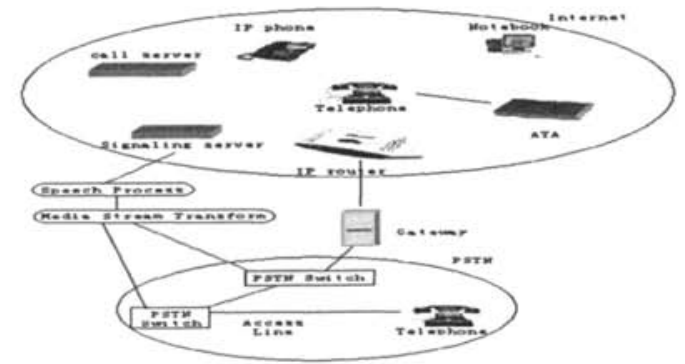

Fig.3 IP-to-PSTN gateway in a VoIP network 
In our system, the gateway connects to a LAN port on the user's IP router, and PSTN access line. It produces the bridge of a voice call between Internet endpoint devices, such as a soft phone. Fig. 3 illustrates the typical position of the gateway. It also includes a call server and a signaling server. A call server is used in the call establishment phase to set up IP communication between an IP device, such as an IP phone, computer, or analogue telephone adapter (ATA) and an IP-to-PSTN gateway. A signaling server may be involved in call establishment if speech process is used.

The system can afford voice-mail communication between the foreign learners and their teacher. Depend on the voice-mail component, the system can send the learners' information which is collected automatically by system to their teacher.

Another important Network Layer Component is Server. Therefore, we apply Java Applet, which dispenses heavy processing burden of the server to the clients. Unlike standard Web pages, which users simply visit and browse, applet-enhanced pages let the learner manipulate applet components and dynamically interact with information, which is distributed into client-side to lessen server's burden.

When applets incorporate large information domains, they begin to resemble complex desktop applications. In an applet, as in an application, learner might have to move between different screens of information that comprise the applet's domain. Each component would have its own view; to access both learners must be able to navigate between the views. It is different than navigating between Web pages, because all applet code resides in the HTML on a single Web page and the browser's navigation tools navigate "between" pages.

\section{System Design and Implementation}

Based on the above design discussion, we design our Pervasive Computing Mandarin e-Learning System as in Fig.4. Java is used due to the isomerism of platform, and JSP used to design the web page.

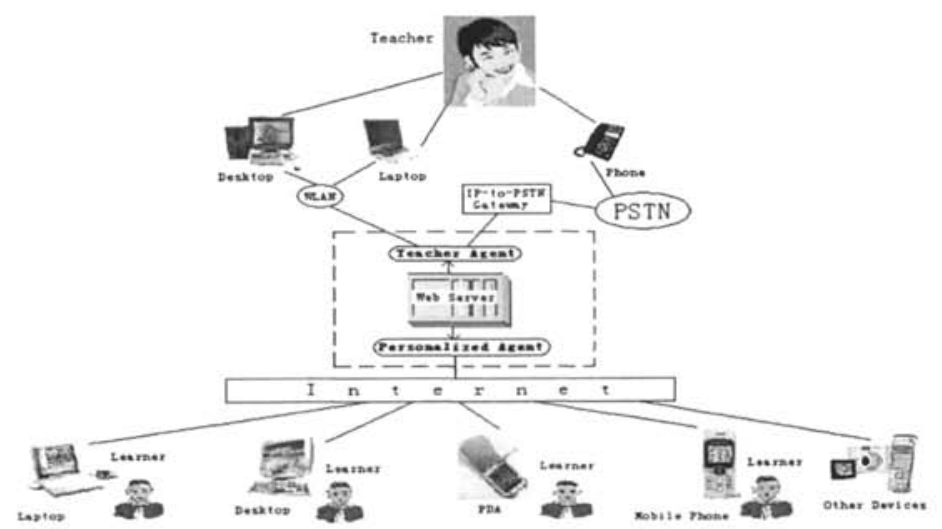

Fig. 4 Pervasive Computing Mandarin e-Learning System 
As one focus is humanizing pedagogy, a major part of our work is to evaluate and analyze learner's language learning activities, and adjust the curriculum contents based on his activity situation. The work process is as follows: firstly, he interacts with the teaching server through Internet; and puts forward his personalized learning requests according to his knowledge structure and learning plan. Then, learner service center analyzes the learner's learning history and demands, creates personalized agent which stands for his learning requests and preferences. Finally, the system distributes the personalized learning services to the learner so that the learner obtains the learning resource or personalized learning guide.

After certain personalized course, a group of test is presented for the learner. His answer is analyzed though personality-analyzing agent. The analysis results are saved in his personalized learning database, which is the basis for the next learning.

This section gives system's implementation discussions. The foreign learner can $\log$ in our e-Learning system by his ID and password through Internet in the Fig.5.

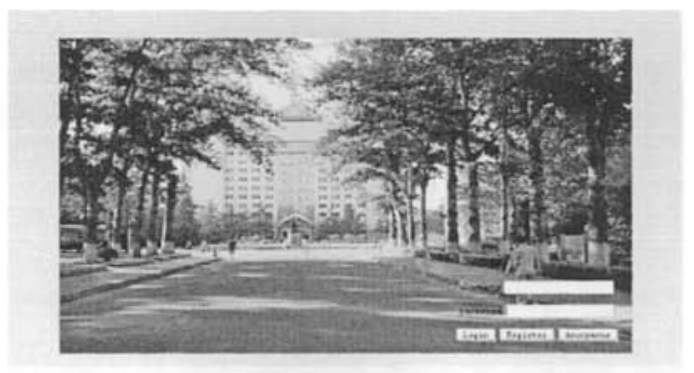

Fig.5 Pervasive Computing Based Mandarin E-learning System Home Page

An example of Mandarin e-learning lessons is shown in Fig.6. For each Mandarin word, we all provide its mean in English, Real-time pronunciation, learner's pronunciation, Speech Recognition and Tone Recognition.

The overall pronunciation of each utterance is rated on a scale of 1-100. In terms of the related theories of speech analysis discussed above, the e-learning system will process the learner's Mandarin speech and the result in Fig.6.

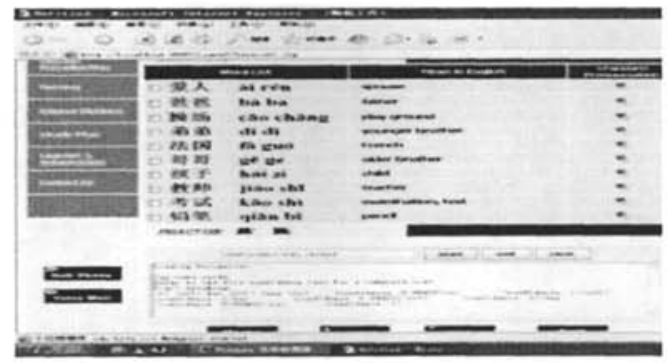

Fig.6 Learner's Pronunciation Exercise Page 


\section{Conclusions}

This paper describes a pervasive computing system design and implementation for Mandarin E-learning. We study its implementation using the LAAD method based on the HPC Model. We analyze the foreign learners' requirements and discuss the implementation in pervasive environment.

Generally the key advantages of our system are: (1) The learner can access our Mandarin e-learning system anytime and anywhere without any restrictions as the system is a pervasive computing based system; (2) It is a humanized learning system which can adjust the learning contents and their presentation formats based on the learner's preferences and his real-time learning states.

This gives us bright hope in the success of our scheme and we are convinced that such a scheme will indeed become practical and scalable for its deployment over Internet for Mandarin e-learning.

\section{Acknowledgments}

This work is supported by National 973 Key Research Program 2006CB303105, National 973 Key Research Program 2004CB318110 and University Key Research Fund 2004SZ002.

\section{References}

1. Z.J. Miao, B.Z. Yuan, M.S. Yu, "A Pervasive Multimodal Tele-Home Healthcare System”, Journal of Universal Computer Science, Vol. 12, No. 1 (2006), 99-114

2. W. Su, Z.J. Miao, "A pervasive Computing System Design and Implementation For Mandarin e-Learning”, The First International Symposium on Pervasive Computing and Applications, Urumchi, China, Aug. 2006, 394-398

3. S. Young, "Large Vocabulary Continuous Speech Recognition: a Review", Cambridge University Engineering Department, 1-23

4. Y. Ming, Z.J. Miao, W. Su, "Tone Analysis for a Pervasive Computing Mandarin e-Learning System", The Second International Symposium on Pervasive Computing and Applications, Birmingham, UK, Jul. 2007

5. Q. Shi, X.J. Ma, W.B. Zhu, W. Zhang, Q.S. Li, "Statistic Prosody Structure Prediction", Speech Technology Group, IBM China Research Lab

6. C. Houser, P. Thornton, and D. Kluge, "Mobile Learning: Cell Phones and PDAs for Education", Computers in Education, 2002. Proceedings International Conference on, Dec. 2002, Vol.2, 1149-1150

7. Q.Y. Gu, T. Sumner, "Support Personalization in Distributed E-learning Through Learner Modeling", Information and Communication Technologies, 2006. ICTTA '06. $2^{\text {nd }}$, Vol 1, 610-615

8. A. E. Conway, "IP Telephone with a Personal IP-PSTN Gateway: Architecture and PSTN-Line Sharing Application", Verizon Laboratories, 132-136 